\title{
A Symptom-based COVID-19 Diagnostic Tool in case of Limited Testing Facilities
}

\author{
R RIDWAN $^{\mathrm{a}}$, MR AMIN $^{\mathrm{b}}$, MR RAHMAN ${ }^{\mathrm{c}}$
}

\begin{abstract}
:
Since December 2019, when a cluster of atypical pneumonia cases were identified in Wuhan, China a new disease has spread across the world. COVID-19 has since become the biggest pandemic in a century, touching lives in almost every country in the world. At the outset of COVID-19, the World Health Organization advised for testing to become a priority so that patients with COVID-19 could be quickly identified, isolated and treated to interrupt transmission of disease. However, testing shortages have been an increasing problem in low and middle income countries. Even when tests are available, it has proved time-consuming. Therefore,
\end{abstract}

Background: Rationale for a Symptom-Based Diagnostic Criteria

Since last week of December 2019, when the first cases of a new atypical pneumonia were described in Wuhan, China, there has been an outbreak of the largest pandemic in a century. Since then, the COVID-19 disease has spread to all corners of the globe with huge morbidity and mortality and brought life to a standstill in many parts of the world. As has been advised by the World Health Organization $(\mathrm{WHO})^{1}$, there is an increasing need to identify patients with suspected COVID-19 symptoms as many as possible by doing test and to try to break the cycle of transmission. However, many hospitals around the world, especially in Low and Middle Income Countries, have struggled to have enough testing kits available to truly understand the caseloads in the country ${ }^{2}$. With plenty of reports of various symptoms that have been reported in current literature $3-6,7-11$, it become prudent that hospitals in Low and Middle

a. Raiiq Ridwan, Specialty Trainee in Emergency Medicine, Cambridge University Hospitals NHS Foundation Trust, UK

b. Md. Robed Amin, Professor of Internal Medicine, Dhaka Medical College Hospital, Dhaka, Bangladesh

c. Md. Ridwanur Rahman, Professor and Head of Research, Universal Medical College Research Centre, Dhaka, Bangladesh

Address of Correspondence: Prof. Md. Robed Amin, Professor of Medicine, Dhaka Medical College, Room no 502, Department of Medicine, Dhaka Medical College.

Email-robedamin@yahoo.com, Cell-01711725787 we propose a symptom-based tool to assist in the diagnosis of COVID-19 management in low and middle income Countries. It is based on the symptoms that have so far been described in the literature and advises the frontline healthcare worker on how to diagnose the likelihood of having COVID-19 and separate the patient into Red (very likely), Yellow (possible) and Green (unlikely) categories.

Keywords: COVID 19 diagnostic tool (CDT), Clinical tool (CT), Low and middle income countries (LMIC).

(J Bangladesh Coll Phys Surg 2020; 38: 71-75) DOI: https://doi.org/10.3329/jbcps.v38i0.47342

Income Countries (LMIC) develop a tool to potentially identify patients with COVID-19 to interrupt transmission and save lives. Moreover, due to the outbreak there has been a policy that telehealth services maybe upscaled for betterment of service and reduction of community transmission ${ }^{7}$. Telehealth practitioners would also need a user-friendly tool to screen patients for possible COVID-19 infection to help identify potential COVID19 patients.

Methods: This one is a system-based exploration study to develop a functional tool for LMIC country. Existing literature on COVID -19 has been searched to develop a model and work for flow development was done in this study. The search was systematic with pubmed and other indexing English literature-based search on COVID-19 and search item includes symptom, diagnostic tool, COVID-19 and literature was search from December 2019 to onwards. The searching was followed by development of workflow based model for easy understanding and with functional component to carry out diagnosis.

\section{Scope of the tool}

The suggestion would be for the tool to be used either in primary care services, emergency departments or in telehealth services to identify patients who may have potential to be a COVID-19 case. While the scoring system does not trump clinical assessment, and other diagnoses are not excluded simply by using this tool, an easy-to-use guide is needed for patients to be triaged to either a COVID-19 or a non-COVID-19 pathway for 
management. Moreover, even in hospitals with some testing facilities, this tool would be helpful in co horting patients to allocating wards based on the likelihood of them having COVID-19. Covid-19 has insidious presentation and is very difficult to rule out simply by clinical judgement. Therefore, this tool is for use in determining "likelihood" of a patient being Covid-19 on presentation to hospital, rather than being a confirmatory

\section{How symptoms were chosen?}

Since the inception, multiple studies ${ }^{3-6,8-11}$ have presented examples of what the patient on presentation have had in terms of symptoms. Table 1 describes the findings of the major symptoms from 6 such studies published recently. These symptomatic patients presented to health departments who later were confirmed to have COVID-19, and looks specifically at the symptoms on presentation

As can be seen from the above table, Fever and Cough were the most common symptoms on presentation. Moreover, there were further symptoms mentioned in various percentage. Notably, multiple studies ${ }^{3,4,11}$ have noted that shortness of breath/dyspnoea would be an indication for urgent referral to hospital.

\section{Guidance on Using the Symptom-Based Tool}

This COVID-19 Likelihood Tool is intended to help clinicians on the frontline in LMIC with limited or no testing facilities. The tool is aimed to help triage patients and advise them based on the likelihood of them having COVID-19 or to admit them to an appropriate hospital. The parameters are based on symptoms that have been reported in current literature. ${ }^{3-6.8-11}$ Primarily, fever and cough are reported as the main symptoms due to being most common symptoms across the course of the disease $^{6}$. However, as seen by Guan et. al, ${ }^{4}$ quite a large percentage of patients may not present with fever on initial presentation. Therefore, contrary to WHO, fever alone could not be a defining character of COVID-19 alone and thus make it important to consider cough as a major symptom as well. The fewer common symptoms are taken as a separate category to help identify COVID19 patients who have either fever or cough or none. The following parameters are to be used

\section{Results and Discussion:}

Symptom based triage need an important red flag territory for admission in health facility and thus the main indication for admission to inpatient hospital is respiratory distress/shortness of breath, as it is associated with severe pneumonia to Acute Respiratory Distress Syndrome (ARDS) and thus poor outcomes $^{3,4,11}$. For the purposes of this tool, and to make it as broadly useful as possible, we would like to say that respiratory distress/shortness of breath would be defined as one of the following symptomatology

Respiratory Rate $>30$ breaths per minute OR

Inability to complete sentences

If Respiratory distress is not present, then the early assessment can move on to diagnosing based on other symptoms.

Table-I

\begin{tabular}{|c|c|c|c|c|c|c|}
\hline \multicolumn{7}{|c|}{ Percentage of patients presenting with various symptoms across different cohorts } \\
\hline & $\begin{array}{c}\text { Guan } \\
\text { et al [4] }\end{array}$ & $\begin{array}{l}\text { Shi e } \\
\text { t al [8] }\end{array}$ & $\begin{array}{c}\text { Chen } \\
\text { et al [9] }\end{array}$ & $\begin{array}{l}\text { Huang } \\
\text { et al [10] }\end{array}$ & $\begin{array}{c}\mathrm{Xu} \\
\text { et al }[11]\end{array}$ & $\begin{array}{l}\text { Yang et al. } \\
\text { (Critically ill } \\
\text { patients) }[11]\end{array}$ \\
\hline Fever & $43 \%$ & $86 \%$ & $83 \%$ & $98 \%$ & $77 \%$ & $88 \%$ \\
\hline Cough & $68 \%$ & $71 \%$ & $82 \%$ & $76 \%$ & $81 \%$ & $77 \%$ \\
\hline Sputum production & $34 \%$ & $14 \%$ & & $28 \%$ & $56 \%$ & \\
\hline Dyspnoea & $19 \%$ & $43 \%$ & $31 \%$ & $55 \%$ & $3 \%$ & $64 \%$ \\
\hline Sore throat & $14 \%$ & & $5 \%$ & & & \\
\hline Myalgia & $15 \%$ & & $11 \%$ & & & $12 \%$ \\
\hline Headache & $14 \%$ & $10 \%$ & $8 \%$ & $8 \%$ & $34 \%$ & $6 \%$ \\
\hline Diarrhoea & $4 \%$ & $5 \%$ & $2 \%$ & $3 \%$ & $8 \%$ & \\
\hline Nausea/Vomiting & $5 \%$ & $10 \%$ & $1 \%$ & & & $6 \%$ \\
\hline
\end{tabular}


Main Symptoms: New FeverAND/OR New Cough 3 3-6,8-11

Less Common Symptoms: Fatigue, Myalgia, Sore throat, Headache, Diarrhoea+/-Vomiting, Anosmia 3-6.8-11

Thereafter, based on the presentation of symptoms, patients can be triaged to the following categories (Figure 1)

COVID Green (very low probability of having COVID19 infection but NOT impossible) - Only one of the Less Common Symptoms with none of the Main symptoms

COVID Yellow (COVID-19 possible but other conditions need to be considered)-Any ONE of the following

1. Presence of ONE main symptom, and none of the less common symptoms

2. Presence of 2 or more of the less common symptoms COVID Red (COVID-19 very likely) - Any ONE of the following
1. Presence of both main symptoms

2. Presence of ONE main symptom AND ONE or more number of less common symptoms

\section{Investigations}

If available and easily attainable in ILMIC country, the following simple investigations in patients could be considered. However, it is important to remember that blood sample handling and CXR rooms could also become a potential site of infection spread, and put healthcare workers at risk. So appropriate IPC measures use and judicious decision of investigation should be practiced.

CXR - shows patchy infiltrates mostly peripheral and basal, or gound glass opacity (about $60 \%$ sensitive) ${ }^{4.8}$ Full Blood Count/Complete Blood Count - Lymphopenia is suggested to be present in about $80 \%$ of patients. ${ }^{4}$

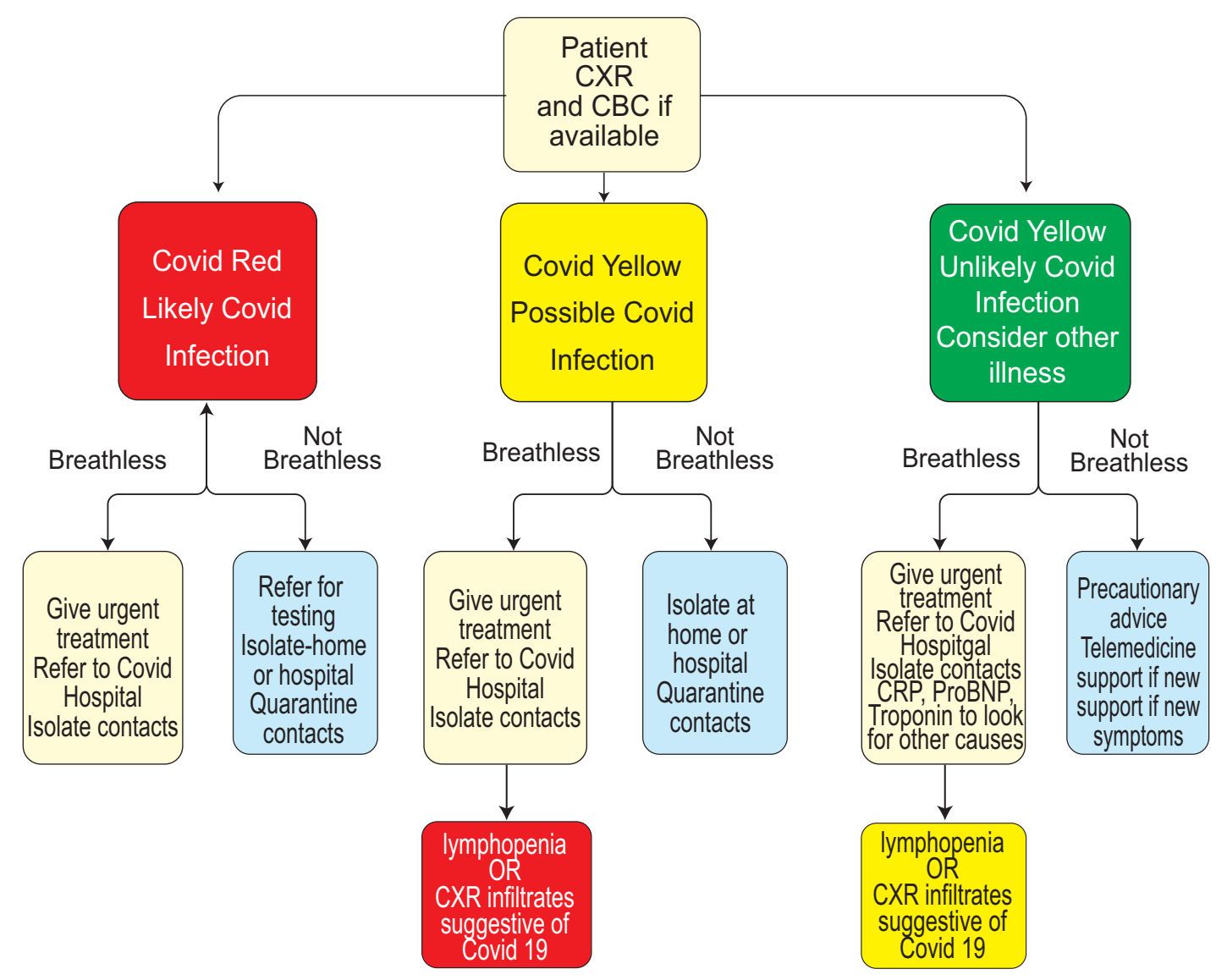

Fig.-1: Covid19 Likelihood Diagnostic Tool 


\section{Pathway for Each Colour-Coded Patient}

After assigning a patient to an appropriate colour code, patients can then be appropriately cohorted based on available facilities. Due to current evidence that patients with shortness of breath may have worse outcomes ${ }^{4.6 .11,12}$, the pathway advises for all patients with shortness of breath to be given urgent treatment and referred to the appropriate hospital for COVID-19 treatment, if available. Moreover, for patients with breathlessness, the pathway suggests doing appropriate investigations if available. There is increasing evidence that Lymphopenia and Chest Xray infiltrates may be diagnostic for COVID-19. . $^{3.6}$ Therefore, any patients who have EITHER of these investigation findings are rated up the pathway. COVID_-Yellow patients would be managed as COVIDRed with investigative findings, and COVID-Green patients as COVID-Yellow. (Figure 2)
Moreover, COVID Green patients who are breathless should be considered for admission to investigate for a wide variety of other conditions that can cause breathlessness. Investigations should typically try to rule out acute causes of breathlessness such as Myocardial Infarction, Acute Heart failure and other systemic causes.

Patients who are not breathless can be managed at home, with appropriate telehealth or written advice, with strict self-home isolation measures in place for COVID Red and Yellow patients. COVID Green patients should be given precautionary advice, and advice to start strict isolation in case of developing of more symptoms.

\section{Limitations}

The authors are aware that this is not currently a validated tool, and validation would only be possible by putting it to the test against the current gold-standard

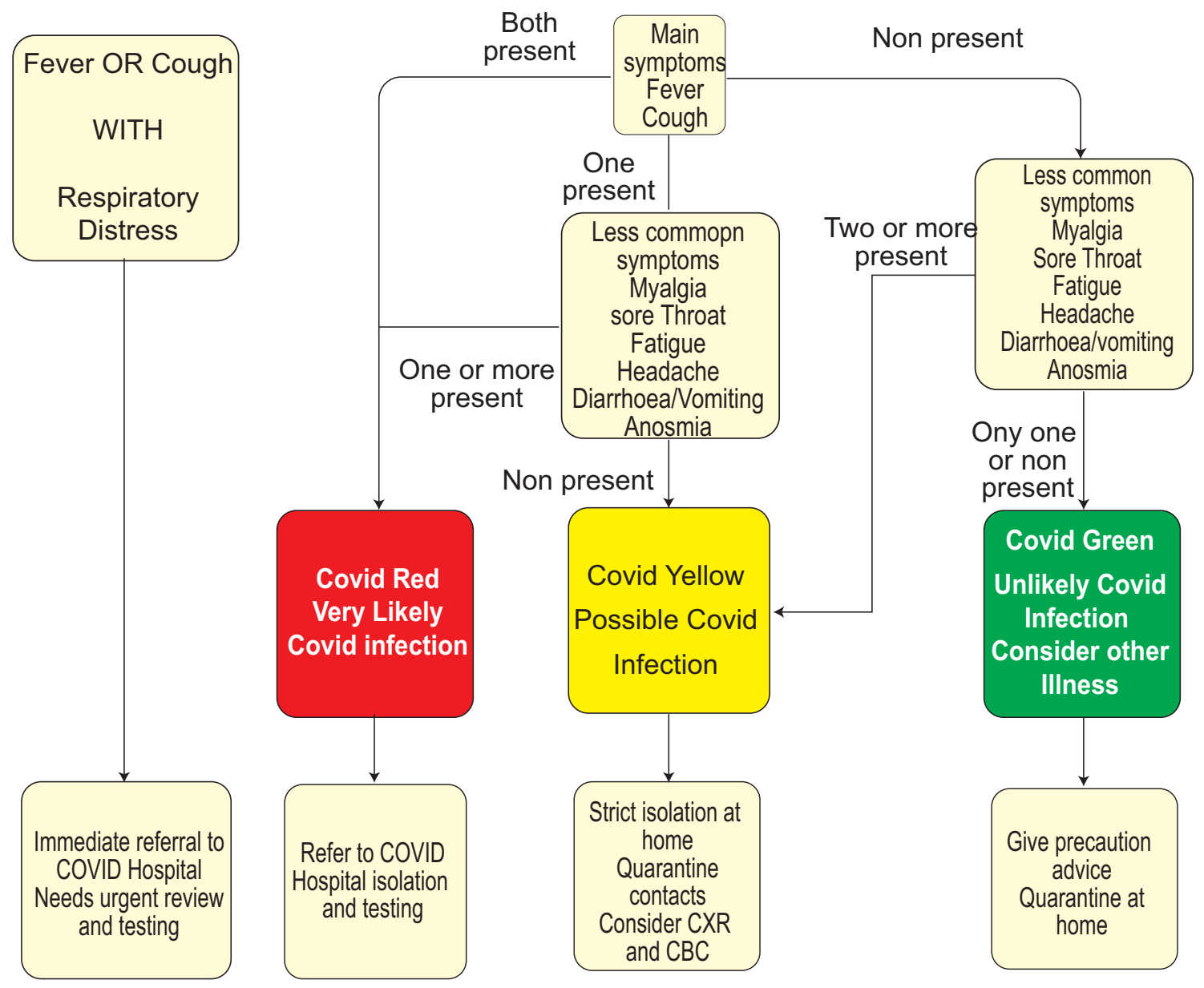

Fig.-2: Pathway for each colour coded patient 
- RT-PCR. However, due to current lack of time and the rapid spread of disease, we are advising for the tool to be used. Moreover, as has been seen in practice, ${ }^{6}$ the sensitivity of RT-PCR from both nasopharyngeal and throat swabs is very low. Therefore, there can be argument made that for COVID-Red patients with a negative PCR test, a repeat swab may be warranted if symptoms continue.

The authors are also aware that the tool may be unable to differentiate between influenza-like illnesses (ILI) and COVID-19 effectively. However, while there is community transmission of a pandemic it is essential that a safety-first approach is taken and hence the diagnostic threshold to be low by clinician. Therefore, the aim was for a high-sensitivity tool, despite the possibility that it could have low-specificity.

\section{Conclusion:}

This Symptom-Based Diagnostic Tool is not meant to replace the gold standard. However, in times of a pandemic, a clinical tool is required to effectively triage, assess and manage patients with the current resource constraints that can be seen. The tool would require further study and the author would welcome more studies on the sensitivity and specificity of the tool. The author would welcome any constructive criticism of the above tool, and any improvement suggestions would be welcome.

\section{References :}

1. WHO Press Bulletin, "You cannot fight a fire blindfolded." $16^{\text {th }}$ March 2020

2. New York Times, Can't Get Tested? Maybe You're in the Wrong Country, $20^{\text {th }}$ March 2020
3. Zhou F., Yu T., Du R. et. al, Clinical course and risk factors for mortality of adult inpatients with COVID-19 in Wuhan, China: a retrospective cohort study Lancet, 395 (2020), pp. 1054-1062 https://doi.org/10.1016/S0140-6736(20) 30566-3

4. Guan, WJ, Ni, ZY, Hu, Y et al. Clinical Characteristics of Coronavirus Disease 2019 in China. $N$ Engl J Med. 2020 DOI: 10.1056/NEJMoa2002032

5. Report of the WHO-China Joint Mission on Coronavirus Disease 2019 (COVID-19), 24/3/2020

6. Thomas-Rüddel, D., Winning, J., Dickmann, P. et al. Coronavirus disease 2019 (COVID-19): update for anesthesiologists and intensivists March 2020. Anaesthesist (2020). https://doi.org/10.1007/ s00101-020-00760-3

7. Hollander JE, Carr BG. Virtually perfect? Telemedicine for COVID-19. N Engl J Med. 2020 https://doi.org/10.1056/ NEJMp2003539

8. Shi H, Han X, Jiang N, et al (2020) Radiological findings from 81 patients with COVID-19 pneumonia in Wuhan, China: a descriptive study. Lancet Infect Dis. https://doi. org/10.1016/S1473-3099(20)30086-4

9. Chen N, Zhou M, Dong X, et al. Epidemiological and clinical characteristics of 99 cases of 2019 novel coronavirus pneumonia in Wuhan, China: a descriptive study. Lancet 2020;395:507-13

10. Huang C, Wang Y, Li X, et al. Clinical features of patients infected with 2019 novel coronavirus in Wuhan, China. Lancet 2020;395:497-506

11. Z Xu, L Shi, Y Wang, et al. Pathological findings of COVID19 associated with acute respiratory distress syndrome Lancet Respir Med (2020) published online Feb 18 https:/ /doi.org/10.1016/S2213-2600(20)30076-X

12. Yang X, Yu Y, Xu J et al. (2020) Clinical course and out comes of critically ill patients with SARS-CoV-2 pneumonia in Wuhan, China: a single-centered, retrospective, observational study. Lancet Respir Med. https://doi.org/ 10.1016/S2213-2600(20)30079-5 